\title{
Patient and Probe Tracking During Freehand Ultrasound
}

\author{
Giselle Flaccavento, Peter Lawrence, and Robert Rohling \\ Department of Electrical and Computer Engineering \\ University of British Columbia, Vancouver, Canada \\ \{peterl, rohling\}@ece.ubc.ca
}

\begin{abstract}
We present a system that measures the probe location with respect to the patient's body during an ultrasound exam. The system uses an inexpensive trinocular camera to track patches of points on a surface. The accuracy of the digital camera system is measured, using the Optotrak as a reference, and is less than $\pm 2 \mathrm{~mm}$ for a $20 \times 20 \mathrm{~mm}$ patch. Fiducials, which can be seen in both the ultrasound images and the camera images, are used for a consistency test producing values with a standard deviation under $3.2 \mathrm{~mm}$.
\end{abstract}

\section{Introduction}

The relative location of ultrasound images is often required for panoramic ultrasound, ultrasound assisted surgery, and freehand 3D ultrasound reconstruction [16]. The ultrasound probe is usually tracked with respect to a fixed coordinate system such as a bed or floor while the patient remains still. This stillness is achieved through the use of breath holds or restraining devices. Patient movement can be involuntary, due to respiration, or due to the force induced by the probe. In the dorsoventral direction, respiratory movement at the navel was recorded as $4.03 \mathrm{~mm}$ [4. Probe force deformation as well as involuntary patient motion can cause much greater movement.

Although sometimes used to reduce the errors caused by respiration, breath holds and respiratory gating are not always feasible solutions as some people, especially young children [15, and sick patients would have difficulties holding their breath. Remaining still may be difficult due to arthritis in the neck, back, or shoulders [5]. Acquiring a set of data for a 3D ultrasound cardiac exam, can take up to $45 \mathrm{sec}$ 11. Maximum patient breath holds have been found to be $41 \pm 20 \mathrm{sec}$ [6. Inconsistencies between breaths and respiratory drift after as little as $12 \mathrm{sec}$ may also occur during a breath hold [9]. As the probe moves along the patient's skin, the tissue is displaced both globally and locally by the force of the probe [14.

In this paper, we present a probe and patient tracking system, shown in Figure 1 for use with both 3D and panoramic freehand ultrasound. We measure the probe location with respect to the patient's body by tracking both the probe and the patient. The goal is to produce a system with an accuracy better than 
the motion incurred during the exam. The system we propose has the ability to track the motion of the area being examined during the ultrasound scan. Although we recognize that there is internal organ movement as well as external patient movement during the acquisition of images, this initial study aims to track only the external patient movement.

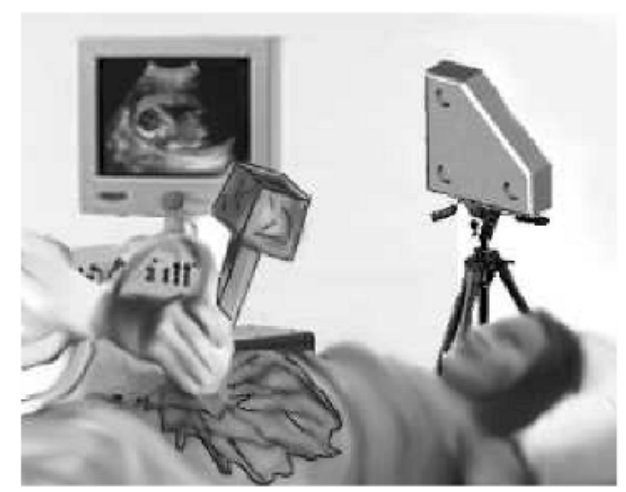

Fig. 1. Complete tracking system composed of both the digital camera and ultrasound tracking components.

Patient and probe motion compensation using external trackers during ultrasound have been previously investigated. In [1, an optical tracking system, with infra-red light emitting diode (IRED) markers, was used to track the probe along with one passive marker to track the navel. A single camera was used in [8] to track a rigid object with 4 known fiducial markers attached. Since only a few markers were used in both of these systems, the line of sight between the sensor and markers must be ensured throughout the procedure [10[13]. In [2], the patient motion is tracked using one magnetic sensor attached to the sternum. Magnetic position trackers are known to have reduced accuracy due to interference from ferromagnetic devices 7 . Instead of tracking the entire surface, these systems track only a small number of points on the patient or probe.

\section{Methods}

For our tracking system, we use a relatively inexpensive trinocular camera to track both the patient and the probe. A small object containing grayscale texture is attached to the probe in order to make tracking possible and more accurate. The number of features on the patient's skin is augmented with a painted grayscale texture as seen in Figure 1 


\subsection{Digital Camera Tracking Component}

The camera tracking component of our system uses a grayscale Digiclops trinocular stereo camera (Point Grey Research Inc., Vancouver, BC), with a field of view of $44^{\circ}$, a $6 \mathrm{~mm}$ focal length, and images of $1024 \times 768$ pixels. The accuracy of the digital camera system is computed in this paper through a series of experiments that track the location of two rigid objects, a calibration flat plate and a calibration sphere, which approximate a probe and the abdomen of a pregnant patient. We use the Optotrak 3020 positioning system (Northern Digital Inc., Waterloo, ON) as a reference standard to calculate the "true" location of our objects during tracking. Before the accuracy of the Digiclops can be calculated, it is first necessary to calculate the origin of the Digiclops coordinate system, $C_{D}$, which is located at the pinhole of one of the cameras, with respect to the Global Optotrak coordinate system, $C_{G}$. This is found by computing the transformation, $T_{G}^{D}$, shown in Figure 2 between $C_{D}$ and $C_{G}$,

$$
T_{G}^{D}=T_{G}^{R} T_{R}^{P} T_{P}^{D}=T_{G}^{R} T_{R}^{P}\left(T_{D}^{P}\right)^{-1}
$$

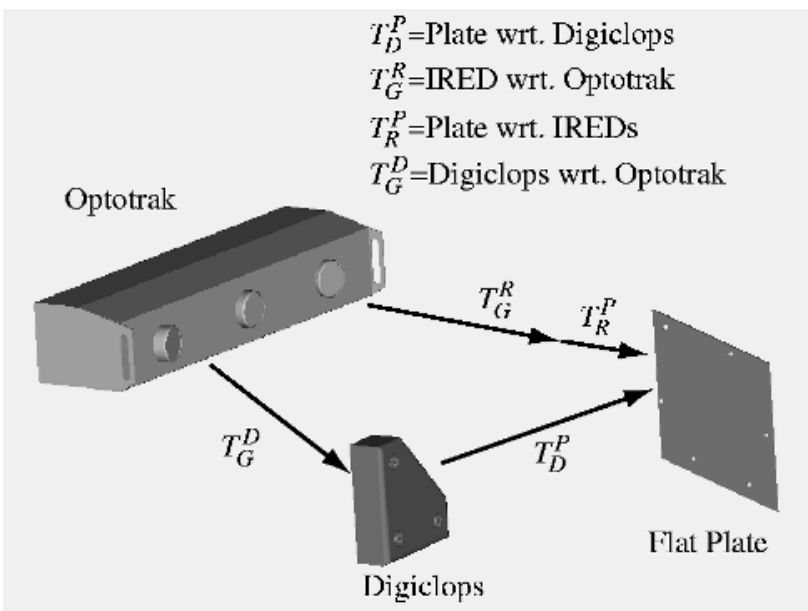

Fig. 2. Transformations used in determining the relationship between the Optotrak and Digiclops coordinate systems.

$\mathbf{T}_{\mathbf{D}}^{\mathbf{P}}$ : The transformation between the plate and the Digiclops, $T_{D}^{P}$, is found using a grayscale texture with non-repeating features overlaid with 14 printed crosses and secured to the plate. The features are manually selected from the Digiclops images. Normalized correlation with subpixel interpolation is used to perform a template match between the two images. The 3D location of each feature point is found using triangulation. A plane and its normal are fitted to the $3 \mathrm{D}$ points using least squares minimization. The plate coordinate system, 


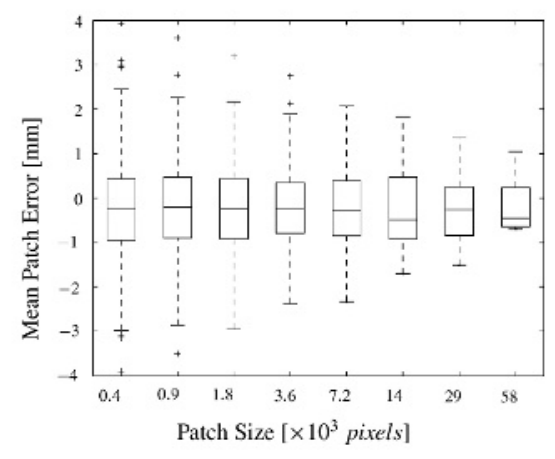

(a) Plate

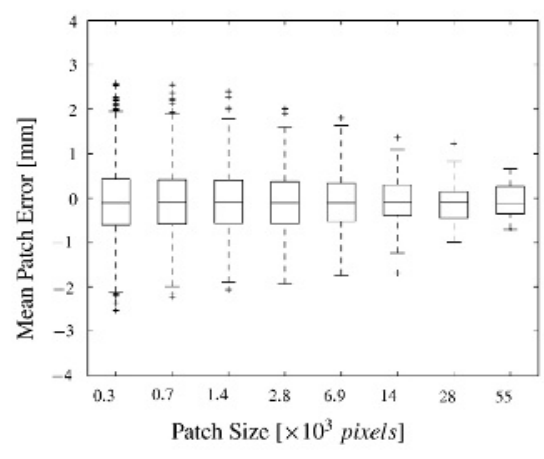

(b) Sphere

Fig. 3. Accuracy of tracking the surface of the flat plate and the sphere using various patch sizes. The standard deviation of the points for each test run is $0.9 \mathrm{~mm}$ to $1.6 \mathrm{~mm}$ for the plate and $0.6 \mathrm{~mm}$ to $1.1 \mathrm{~mm}$ for the sphere.

$C_{P}$, has a $y$ direction that is computed by finding the direction vector between two chosen $3 \mathrm{D}$ points in the plane. The $z$ direction is calculated parallel to the normal of the plane and passing through the origin of $C_{P}$. Finally, the $x$ direction is calculated as the cross product of the $y$ and $z$ direction vectors. These vectors are then used to create $T_{D}^{P}$.

$\mathbf{T}_{\mathbf{G}}^{\mathbf{R}}$ : Six IREDs are attached to the surface of the plate enabling the Optotrak to also measure the plate's location. The transformation, $T_{G}^{R}$, from the IRED coordinate system, $C_{R}$, to $C_{G}$ is next found. The geometry between 3 IREDs, secured to the back of the Digiclops case, is used to define $C_{G}$. Using the method described previously to find $T_{D}^{P}, T_{G}^{R}$ is calculated.

$\mathbf{T}_{\mathbf{R}}^{\mathbf{P}}$ : The transformation, $T_{R}^{P}$, describes the offset between the location where the IREDs are attached to the plate and their recorded location based on the calibrated LED thickness.

$\mathbf{T}_{\mathbf{G}}^{\mathrm{D}}$ : Data is collected using various plate to Digiclops distances and angles. After calculating $T_{G}^{R}, T_{R}^{P}$, and $T_{D}^{P}$ for each run, it is possible to calculate the unknowns in $T_{G}^{D}$ using a least squares minimization to solve Equation [1.

The accuracy of the Digiclops system is next tested. A grayscale texture and 6 IREDs are attached to a flat test plate. The surface of a test sphere is painted with a grayscale texture and 12 IREDs are attached. The plate and sphere are placed at various locations ranging from $860 \mathrm{~mm}$ to $1050 \mathrm{~mm}$ from the Digiclops camera in order to mimic the workspace in a clinical setting. A Digiclops window of $360 \times 160$ pixels for the plate images and $240 \times 230$ pixels for the sphere images, records the $3 \mathrm{D}$ location of all detected features. The Optotrak records the IRED locations, $P_{G}$, which are next transformed from $C_{G}$ to $C_{D}$ using $\left(T_{G}^{D}\right)^{-1}$. 
The error between the points calculated with the Digiclops and the true surface location for the plate and sphere are calculated as follows:

$$
\begin{gathered}
\text { error }_{\text {plate }}=\frac{A \cdot P_{D_{x}}+B \cdot P_{D_{y}}+C \cdot P_{D_{z}}+D}{ \pm \sqrt{A^{2}+B^{2}+C^{2}}} \\
\text { error }_{\text {sphere }}=\sqrt{\left(P_{D_{x}}-P_{D, \text { cen }}\right)^{2}+\left(P_{D_{y}}-P_{D, \text { cen }}\right)^{2}+\left(P_{D_{z}}-P_{D, \text { cen }}\right)^{2}}-r_{\text {true }}
\end{gathered}
$$

where $P_{D}\left(P_{D_{x}}, P_{D_{y}}, P_{D_{z}}\right)$ is each point recorded by the Digiclops, $A x+B y+$ $C z+D=0$ is the true flat plate, and $P_{D}\left(P_{D, c e n_{x}}, P_{D, \text { cen }_{y}}, P_{D, c e n_{z}}\right)$ and $r_{\text {true }}$ are the true centre and radius of the sphere.

Our system obtains its accuracy by using the mean of a large number of point locations within each patch to calculate the surface location. The effect of accuracy on the size of each patch is therefore computed and the results are shown in Figure 3

\subsection{Image Based Consistency Test}

The results found in subsection 2.1 show that using the Digiclops in our tracking system is feasible as it has sufficient accuracy for tracking large patient motion. An experiment is conducted to assess the consistency of the system when both probe and patient tracking are combined. A flat plate with a textured surface is attached to the probe, creating a surface with features that can be tracked by the Digiclops. The plate to the ultrasound imaging plane must also be located by calibration [12]. N-shaped fiducials are attached to the skin surface to assist in tracking. The components of this experiment create a mock scenario of the complete tracking system and are shown in Figure 4

In this experiment, the patient is represented by one of two life-size tissue mimicking phantoms that approximate the acoustic properties of soft human tissue, while remaining rigid under the force of the probe. Two casts are first created from human models using plaster embedded gauze. One model is made from the torso of a 39 week pregnant female and the second from an adult male. A solution of distilled water and $8 \%$ ( $\mathrm{vol}$.) glycerol, is mixed with $3 \%$ (mass) cellulose particles, and $3 \%$ (mass) agar. The mixture is heated to $85^{\circ} \mathrm{C}$, cooled to $60^{\circ} \mathrm{C}$, and poured into the torso casts.

Overlaid on the surface of the patient is an artificial skin made of latex and containing $\mathrm{N}$-shaped fiducials. Latex was chosen as a matrix material as it does not alter the ultrasound image while the $\mathrm{N}$ fiducials produce small bright spots near the top of the ultrasound image. Using a similar approach to 312 , the distance between the bright spots as well as their widths provide enough information to calculate the unique probe location with respect to each fiducial. For this experiment, only two $\mathrm{N}$ shaped fiducials are needed. In our experiment, one is visible to the Digiclops and the other in the ultrasound image. After extensive testing of different materials, soldered pieces of $1.24 \mathrm{~mm}$ diameter steel sewing needles were used to create the fiducials. The fiducials are placed 


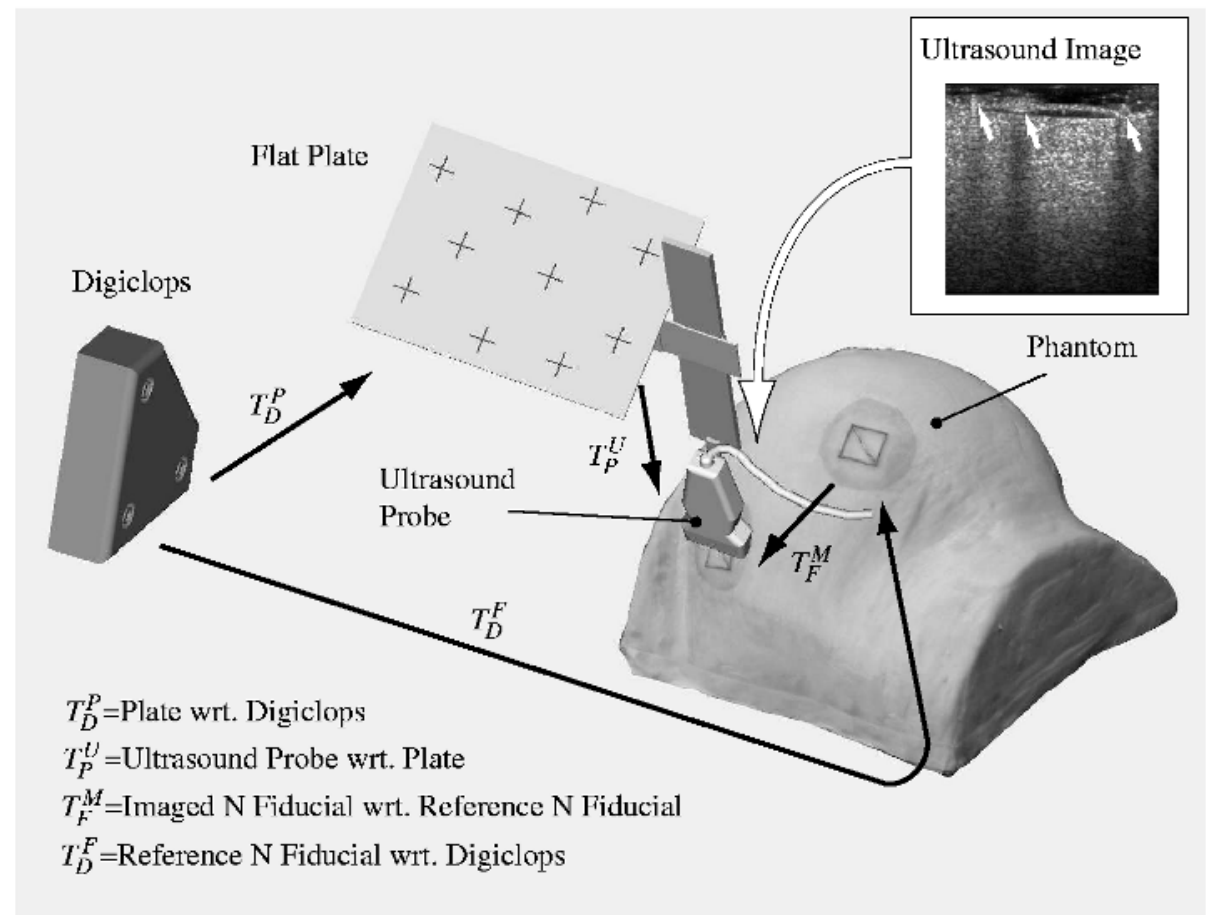

Fig. 4. Relationship Between the Transformations used for the Tracking Experiment. An example ultrasound image is shown with arrows indicating the bright spots created by the $\mathrm{N}$ fiducial.

on moulds that have the form of each phantom. Several coats of latex rubber are applied to the surface of each mould, and then cured in a vacuum to remove any air bubbles.

In order to investigate the consistency of the results, data that describes the location of the ultrasound probe in $C_{D}$ is calculated using two different sets of transformations. The points are transformed from the imaged fiducial coordinate system, $C_{M}$, and the ultrasound coordinate system, $C_{U}$,

$$
P_{D_{I}}=T_{D}^{F} T_{F}^{M} P_{M}, \quad P_{D_{I I}}=T_{D}^{P} T_{P}^{U} P_{U}
$$

and the error is calculated between the points, $P_{D_{I}}$ and $P_{D_{I I}}$, and is shown in Table 1 .

$\mathbf{P}_{\mathbf{M}}, \mathbf{P}_{\mathbf{U}}$ : The centre and diameter of each bright spot in the ultrasound image as seen in Figure 4 are manually located in $C_{U}$ and a unique probe location is calculated within $C_{M}$. The chosen centres of the bright spots in $C_{U}$ and calculated corresponding points in $C_{M}, P_{U}$ and $P_{M}$, provide the starting points for the completion of the loop. 
$\mathbf{T}_{\mathbf{P}}^{\mathbf{U}}$ : The probe is calibrated, providing the transformation between the ultrasound image and the plate, $T_{P}^{U}$, using a variation of the method presented in 12 .

$\mathbf{T}_{\mathbf{D}}^{\mathbf{F}}, \mathbf{T}_{\mathbf{F}}^{\mathbf{M}}, \mathbf{T}_{\mathbf{D}}^{\mathbf{P}}: \quad$ The Digiclops is used to find the transformation $T_{D}^{F}$, between the reference fiducial coordinate system, $C_{F}$, and $C_{D}$, using the same equations used to find $T_{D}^{P}$. The transformation $T_{F}^{M}$ from $C_{M}$ to $C_{F}$ is made possible because the two $\mathrm{N}$ fiducials are fixed relative to each other during this experiment.

Table 1. Error between the points $P_{D_{I}}$ and $P_{D_{I I}}$ using 40 sets of probe locations on the phantom torso.

\begin{tabular}{|l||l|l|l|}
\hline & $\mathbf{X}_{\mathbf{D}}$ direction & $\mathbf{Y}_{\mathbf{D}}$ direction & $\mathbf{Z}_{\mathbf{D}}$ direction \\
\hline \hline Mean $[\mathbf{m m}]$ & -0.1 & -0.3 & -1.1 \\
\hline Maximum [mm] & 6.7 & 8.3 & 2.4 \\
\hline Standard Deviation $[\mathbf{m m}]$ & 2.9 & 3.2 & 1.7 \\
\hline
\end{tabular}

\section{Discussion}

From Figure 3 we see that the Digiclops system is able to track a flat and spherical rigid object with mean errors less than $\pm 1.0 \mathrm{~mm}$ and $\pm 0.7 \mathrm{~mm}$, respectively. Using the mean of 58000 pixels for the plate and 55000 pixels for the sphere, the standard deviations for the points are less than $1.6 \mathrm{~mm}$ and $1.1 \mathrm{~mm}$ for the plate and sphere, respectively. The location error for both the plate and the sphere tests remain under $\pm 2 \mathrm{~mm}$ as the patch size decreases to under 400 pixels. This is equivalent to an area of $20 \times 20 \mathrm{~mm}$ on the surface of the patient's skin. At a distance of $1000 \mathrm{~mm}$, the Digiclops is able to image an area of $600 \times 800 \mathrm{~mm}$. As a comparison of accuracies, the Fastrak ${ }^{\circledR}$ A/C magnetic tracker (Polhemus Inc., Colchester, VT) has an RMS accuracy of $0.762 \mathrm{~mm}$, the Flock of Birds ${ }^{\circledR} \mathrm{D} / \mathrm{C}$ magnetic tracker (Ascension Technology Corp., Burlington, VT) has an RMS accuracy of $1.8 \mathrm{~mm}$, and the Optotrak has an RMS accuracy of $0.15 \mathrm{~mm}$. These systems track few points at a time as well as inhibit the ultrasound probe from passing over the area being tracked since the markers are protruding from the skin surface.

The consistency test reported a maximum mean error for each of the directions of less than $1.1 \mathrm{~mm}$ with a standard deviation under $3.2 \mathrm{~mm}$. Errors during this second experiment are due to the variation in manual selection of the bright spots in the ultrasound image, feature mismatching between the stereo images, and probe calibration errors. As an approximation to the error introduced by the calibration procedure, a similar method, described in [12, resulted in a mean error of $0.23 \mathrm{~mm}$ with a standard deviation of $2.89 \mathrm{~mm}$. Regardless of which external tracking system is used, a calibration between the probe and images is necessary and will introduce errors. For our system, this calibration error is included in our results. The consistency tests inspired ways to reduce this error. 
We propose that the $\mathrm{N}$ fiducials be used to make corrections to the location measurements. We first estimate the location from the cameras and then correct this estimation using the 3 bright spots in the ultrasound image. This correction is made possible because the location of each fiducial is known relative to the grayscale surface. The main goal is to find the ultrasound image with respect to the skin. So, the incorporation of $\mathrm{N}$ fiducials into our tracking system could create on-the-fly correction of the ultrasound image location with respect to the skin.

We envision the camera and ultrasound components of our system working together to ameliorate the accuracy of the entire tracking system. In addition to improving accuracy, the complete system could be able to compensate for occlusions from the camera system. The camera tracking system is necessary to identify which particular fiducial is being imaged as well as to find the angles between the yaw and pitch of the probe. Unlike the feasibility experiment described in this paper, the complete system would be composed of many $\mathrm{N}$ fiducials. Although the surface is covered with fiducial marks, the width and material of the fiducials and matrix are such that a bright dot appears at the top of the ultrasound image but the anatomical information contained in the image is compromised very little. The complete system would also have a smaller object attached to the probe instead of the larger plate that we used in this experiment.

\section{Conclusions}

The accuracy of the inexpensive trinocular camera system that is used to track a patch of $20 \times 20 \mathrm{~mm}$ is less than $\pm 2 \mathrm{~mm}$. A plate was attached to the probe and a textured surface was placed on the skin surface. A consistency test of the complete system produced values with a standard deviation under $3.2 \mathrm{~mm}$. These errors include all calibration steps required for a working system, and are sufficiently small to allow compensating of medium to large patient motion.

Future research with this tracking system will focus on implementing the error correction from multiple $\mathrm{N}$ fiducials to compensate for even smaller patient motion. Automation of bright spot detection in the ultrasound images as well as fiducial detection in the camera images must also be implemented. Studies into local deformation are also planned with camera tracking of small patch sizes.

\section{References}

1. D. Atkinson, M. Burcher, J. Declerck, and J. Noble. Respiratory motion compensation for 3-D freehand echocardiography. Ultrasound Med Biol, 27(12):1615-1620, 2001.

2. M. L. Chuang, M. G. Hibberd, R. A. Beaudin, M. G. Mooney, M. F. Riley, J. T. Fearnside, and P. S. Douglas. Patient motion compensation during transthoracic 3-D echocardiography. Ultrasound Med Biol, 27(2):203-209, 2001. 
3. R. M. Comeau, A. F. Sadikot, A. Fenster, and T. M. Peters. Intraoperative ultrasound for guidance and tissue shift correction in image-guided neurosurgery. Med Phys, 27(4):787-800, 2000.

4. A. DeGroote, M. Wantier, G. Cheron, M. Estenne, and M.Paiva. Chest wall motion during tidal breathing. J Appl Physiol, 83(5):1531-1537, 1997.

5. D. D. Dershaw. Imaging guided biopsy: An alternative to surgical biopsy. Breast $J, 6(5): 294-298,2000$.

6. R. Groell, G. J. Schaffler, and S. Schloffer. Breath-hold times in patients undergoing radiological examinations: Comparison of expiration and inspiration with and without hyperventilation. Radiol and Oncol, 35(3):161-165, 2001.

7. A. Hartov, S. Eisner, M. David, W. Roberts, K. Paulsen, L. Platenik, and M. Miga. Error analysis for a free-hand three-dimensional ultrasound system for neuronavigation. Neurosurg Focus, 6(3), 1999.

8. M. Lee, N. Cardinal, and A. Fenster. Single-camera system for optically tracking freehand motion in 3D: Experimental implementation and evaluation. In Proceedings SPIE Visualization, Display and Image-guided Procedures, pages 109-120, 2001.

9. K. McLeish, D. L. Hill, D. Atkinson, J. M. Blackall, and R. Razavi. A study of the motion and deformation of the heart due to respiration. IEEE T Med Imaging, 21(9):1142-1150, 2002.

10. D. M. Muratore and J. Robert L. Galloway. Beam calibration without a phantom for creating a 3-D freehand ultrasound system. Ultrasound Med Biol, 27(11):1557$1566,2001$.

11. T. R. Nelson and D. H. Pretorius. Three-dimensional ultrasound imaging. Ultrasound Med Biol, 24(9):1243-1270, 1998.

12. N. Pagoulatos, D. R. Haunor, and Y. Kim. A fast calibration method for 3d tacking of ultrasound images using a spatial localizer. Ultrasound Med Biol, 27(9):1219-1229, 2001.

13. G. Penney, J. Blackall, M. Hamady, T.Sabharwal, A.Adam, and D. Hawkes. Registration of freehand 3D ultrasound and magnetic resonance liver images. Med Image Anal, 8(1):81-91, 2004.

14. R. W. Prager, A. Gee, and L. Berman. Stradx: Real-time acquisition and visualization of freehand three-dimentional ultrasound. Med Image Anal, 3(2):129-140, 1998.

15. M. Riccabona, G. Fritz, and E. Ring. Potential applications of three-dimensional ultrasound in the pediatric urinary tract: Pictorial demonstration based on preliminary results. Eur Radiol, 13(12):2680-2687, 2003.

16. A. Roche, X. Pennec, G. Malandain, and N. Ayache. Rigid registration of 3-D ultrasound with $\mathrm{mr}$ images: A new approach combining intensity and gradient information. IEEE T Med Imaging, 20(10):1038-1049, 2001. 